\title{
Robustness of the Tokamak Error Field Correction Tolerance Scaling
}

\author{
N.C. Logan ${ }^{1}$, J.-K. Park ${ }^{1}$, Q. Hu ${ }^{1}$, C. Paz-Soldan ${ }^{2}$, T.

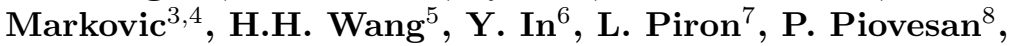 \\ C.E. Myers ${ }^{1} \ddagger$, M. Maraschek ${ }^{9}$, S.M. Wolfe $^{10} \S$, E.J. Strait ${ }^{2}$, \\ S. Munaretto ${ }^{2}$ \\ ${ }^{1}$ Princeton Plasma Physics Laboratory, Princeton, New Jersey 08540, USA \\ 2 General Atomics, San Diego, California 92186, USA \\ ${ }^{3}$ Institute of Plasma Physics of the Czech Academy of Sciences, Czech Republic \\ ${ }^{4}$ Faculty of Mathematics and Physics, Charles University in Prague, Czech \\ Republic \\ ${ }^{5}$ Institute of Plasma Physics Chinese Academy of Sciences, China \\ ${ }^{6}$ Ulsan National Institute of Science and Technology, Korea \\ 7 Culhan Centre of Fusion Energy, UK \\ 8 Consorzio RFX, Italy \\ 9 Max Planck Institute of Plasma Physics, Germany \\ 10 Plasma Science and Fusion Center, Massachusetts Institute of Technology, \\ Cambridge, Massachusetts 02139, USA \\ E-mail: nlogan@pppl.gov \\ June 11, 2020
}

\begin{abstract}
This paper presents the subtleties of obtaining robust experimental scaling laws for the core resonant error field threshold that leads to field penetration, locked modes, and disruptions. Recent progress in attempts to project this threshold to new machines has focused on advances in the metric used to quantify the dangerous error fields, incorporating the ideal MHD plasma response in a metric referred to as the "dominant mode overlap". However, the scaling of this or any quantity with experimental parameters known to be important for the complicated tearing layer physics requires regressions performed for databases that, for historical reasons, unevenly sample the available parametric space. This paper presents the distribution of the existing international $n=1$ database and details biases in the available sampling and details the sensitivity of ITER projections to simple least-squares regressions. Downsampling and a simple kernel density estimation weighted regression are used here to demonstrate the difference in projections that acknowledging the machine sampling bias can make. This results in more robust projection to parameters far from the "usual" devices built thus far. Two multi-device and multi-parameter scalings of the EF threshold in Ohmic and powered plasmas are presented, projecting the threshold to ITER and investigating the impact of sampling biases.
\end{abstract}

Submitted to: Plasma Phys. Control. Fusion

$\ddagger$ Present Address: Sandia National Laboratories, Albuquerque, New Mexico 87185, USA

$\S$ Retired 


\section{Error Field Thresholds Scaling}

Small non-axisymmetric magnetic fields known as error fields (EFs) cause tearing modes to grow and lock in tokamaks, causing disruptions. In all tokamaks, which are designed to be nominally axisymmetric devices, some unavoidable level of construction asymmetry creates "intrinsic" EFs. Even intrinsic EFs that are much smaller (typically on the order of $10^{4}$ times smaller) than the axisymmetric fields are capable of causing disruptions. The critical question in each device is not whether a small EF exists, but how small of an EF is small enough.

Realizing the critical role EFs can play in limiting tokamak performance, devices such as COMPASS [1], DIII-D [1-5], EAST [6], Alcator C-Mod [7], JET [8], NSTX [9], ASDEX-Upgrade [10], KSTAR [11] and others have devoted considerable resources to quantifying and correcting their intrinsic EFs. These experiments recognized that it is critical to the success of their device that the low toroidal mode number components of the asymmetric EF be below a threshold at which they lead to lock modes, and set out to find that threshold. Additional resources have been devoted to studying parametric dependencies of this threshold in individual devices [7-9, 12-20] and comparing these trends with reduced theoretical predictions [21-24]. This effort has provided a solid physics basis for understanding of EF penetration and locking, which enables better predictions of where EFs are most dangerous (i.e. low density, low rotation). Still, the rich physics of the nonlinear resistive tearing layer has made quantitative model prediction of the threshold in new scenarios difficult. As such, the community has developed multi-device, multiparameter scalings for the projection of thresholds to new devices [25-27].

Modern empirical scaling projections to ITER use a threshold metric known as the "dominant mode overlap" (detailed extensively in Refs. [5, 28-30]), which takes into account the well known physics of the ideal magnetohydrodynamic (MHD) plasma response to external EFs. This overlap parameter, $\delta$, is fit to a multi-parameter power law scaling function, $\delta=$ $10^{\alpha_{c}} \prod_{i} p_{i}^{\alpha_{i}}$, where $p_{i}$ are empirical parameters known to influence the threshold and the exponents $\alpha_{c}$ and $\alpha_{i}$ are free parameters of the fit. Note, the overlap is a dimensionless parameter that can be thought of as the ratio of dangerous EF to the axisymmetric field. Such scalings have provided predictions for the threshold in the ITER device before its operation, influencing its strict construction tolerances and EF correction coil designs [25, 31, 32].

When developing a scaling law for extrapolation of observed physics to new devices, the broadest possible trends are desired. The multi-device confinement scalings for example, smooth over the details of local behaviors in individual machines in favor of a broad description of confinement times across many machines and many orders of magnitude in the relevant parameters [33]. Empirical scalings of the EF penetration threshold aim to provide a similarly broad and robust estimation of the critical EF to guide the design and optimization of future devices that will be operated in a hitherto unexplored parametric space. In order to do so, however, the fit scalings must be careful to avoid sensitivities to uneven or correlated distributions of the empirical data available for these regressions. Despite extensive use of scaled threshold in the ITER EF correction strategy and coil design [25, 31, 32, 34-36], neither an in-depth description of the database distribution used to determine these thresholds nor a study of the sensitivity of the projection to particular data has yet been published.

The remainder of this paper details the data and scaling sensitivities in the existing ITPA database of empirical EF threshold data used to make projections to ITER. It demonstrates the robustness of the existing ITER scalings to permutations of the data and compares the standard least-squares regression to an alternate scheme that better matches the most unique data on the near-ITER boundaries of the explored parametric space.

\section{Distribution of Available Data}

The International Tokamak Physics Activity (ITPA) magnetohydrodynamics (MHD) topical group began a joint experiment "MDC-19: Error Field Control at Low Plasma Rotation" in 2012 that has since overcome many challenges to provide error field threshold projections to ITER. In the last five years, three similar-size tokamak devices (DIII-D, EAST, and KSTAR) have contributed to the database with extensive error field (EF) threshold studies documenting their intrinsic EF as well as the scaling of thresholds in applied EFs using advanced 3D field coil systems. This has resulted in an abundance of 


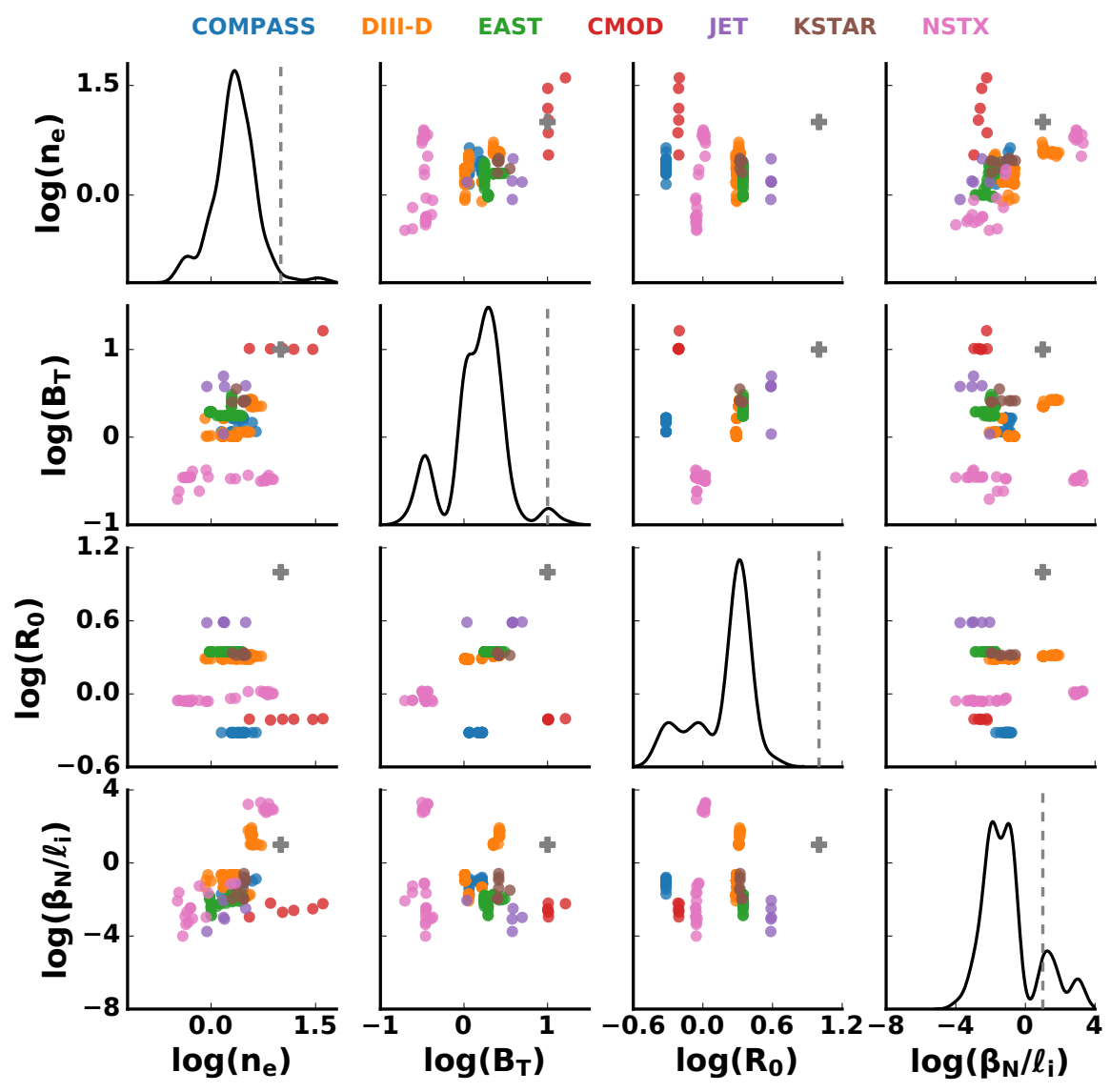

Figure 1. Distribution of the experimental $n=1 \mathrm{EF}$ threshold database across the regression parameters of density, toroidal field, major radius, and normalized pressure. Single-parameter KDEs are plotted in axes along the diagonal to elucidate the uneven sampling densities. Individual points are color coded by device according to the top legend. ITER scenario values are shown as grey "+" symbols and dashed lines.

data in relatively similar plasma sizes, densities, and toroidal fields. During this time, machines like NSTX, JET and C-MOD with vastly different size and toroidal field focused on new priorities or where shut down. Of course, all the devices built to date have been smaller and lower in at least some of the vital parameters (e.g. density, temperature, and confinement) than a fusion reactor envisioned along the lines of ITER and DEMO. Thus, the available EF threshold data is unevenly distributed across the relatively limited parametric space accessible by current devices while scalings using this data are desired for projections outside of its domain.

Figure 1 shows the distribution of the empirical $n=1$ ( $n$ is the toroidal mode number) EF thresholds across four typical regression parameters for 177 plasmas. These plasmas comprise the latest ITPA database used in Ref. [27], including the same additional EAST, DIII-D and COMPASS data and excluding the same cases with significant non-resonant $\mathrm{EF}$ effects to ensure the focus is on the resonant EF physics we are trying to capture. The log quantities are plotted in figure 1 , as this provides the most intuition for the power scaling fit (log linear regression). In addition, all log quantities have been divided by the $\log$ of the corresponding ITER parameter. Projected ITER scenario parameters from Ref. [37] are used here and throughout this paper. They are, $n_{e} \approx$ $9.8 \times 10^{19} \mathrm{~m}^{-3}, B_{T} \approx 5.3 T, R_{0} \approx 6.2 \mathrm{~m}$, and $\beta_{N} / \ell_{i} \approx$ 1.8. Throughout this work, the line average density $n_{e}$ has units of $10^{19} \mathrm{~m}^{-3}$, the on-axis toroidal field $B_{T}$ has units of Tesla, the major radius $R_{0}$ has units of meters and the normalized pressure $\beta_{N} / \ell_{i}$ is unitless. This normalized log space provides a sense of how well our database constrains the log linear power scaling regression around ITER-like parameters (i.e. unity on each axis).

The axes along the diagonal of this figure 1 show kernel density estimates (KDEs) of the corresponding single-parameter probability density function (PDF) across all machines of the database. These curves provide a continuous estimate of the PDF from the 
data, peaking where sampling is heavily sampled and falling where the data is sparse. In order to better visualize the relative density across the database range, the amplitudes are independently scaled to fill the available axes. It is the shape, not the amplitude, that is of interest here. The top left KDE shows that the density sampling is quite concentrated below ITER values, with only a relatively small tail at higher densities. The toroidal field data has more of a bior tri-modal distribution, and also just marginally encompasses the ITER value. Of course, the available data does not extend to ITER values of major radius. However, the data is relatively evenly distributed in the $\log$ radius other than the concentration of data from DIII-D, EAST, and KSTAR near $R_{0} \approx 1.7$. Finally, the normalized pressure is sampled in a bimodal distribution of low $\beta_{N} / \ell_{i}$ Ohmic or L-mode discharges and high $\beta_{N} / \ell_{i}$ H-modes (well surpassing the chosen ITER scenario projection).

The kernel density distributions shown here highlight key gaps in the database that can be filled. One such gap is found in the toroidal field values between NSTX and DIII-D. There is an opportunity for NSTX-U to fill this gap soon when it begins operation. Similarly, there is an opportunity for future compact, high-field devices to supplement the limited data near the high ITER field values. In addition to the opportunities for new devices, the database distribution clearly reveals that there is a valuable opportunity for all existing devices to contribute meaningful data to this ITPA effort in high normalized pressure plasma scenarios.

The off diagonal axes show pair-wise scatter plots of the individual data points from each machine. A larger range is preferable for a well constrained regression, as is a dense sampling of the entire domain (i.e. a flatter KDE). These pair plots show the ITERlike density and toroidal field samples both come from a single C-MOD density scan experiment. They also show that DIII-D and NSTX are the only devices to have contributed data from high $\beta_{N} / \ell_{i} \mathrm{H}$-modes.

Importantly, these pair plots also reveal correlations between each of the parameters used in the regression. The density and pressure are highly correlated. The toroidal field and density are also highly correlated. These are natural consequences of the Ohmic plasmas that make up the majority of the database. However, these couplings do have the potential to influence the ultimate regression. In the worst case, tight co-linearity could increase the condition number of the fit basis far above unity and enable exponent mixing (the ability to arbitrarily increase one exponent while decreasing another without changing the fit error). Although Section 3 shows the situation in this case is not as dire as arbitrary mixing, care must
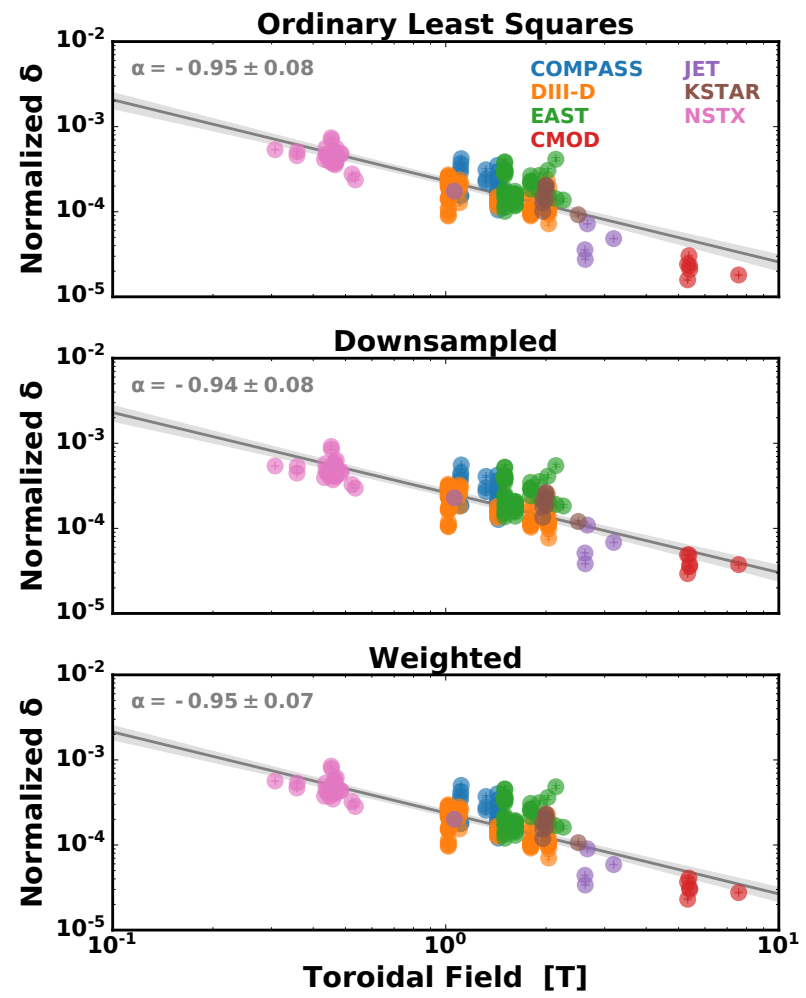

Figure 2. Empirical EF thresholds normalized by $\left[n_{e}^{\alpha_{n}} R_{0}^{\alpha_{R}}\left(\beta_{n} / \ell_{i}\right)^{\alpha_{\beta}}\right]$ as a function of toroidal field for the ordinary least squares (top), downsampled least squares (middle) and weighted least squares (bottom) regression. In each case, a grey line shows the fit toroidal field scaling, and all other $\alpha$ 's can be found in Table 1.

be taken to obtain reliable fits for this heavily biased and coupled data.

This insight should be used to guide new experiments that purposefully decouple these parameters. This can be done using different mixes of electron cyclotron heating $(\mathrm{ECH})$ and neutral beam injection (NBI) heating, for example. Such experiments would improve the condition number (and thus have high impact on the ultimate uncertainty) of the regressions.

\section{Regression}

This section details a relatively simple, multi-machine, multi-parameter power law scaling of the EF threshold. The scaling is shown to be robust, providing confidence in the threshold values previously used for ITER studies.

\subsection{Ordinary Least Squares Regression}

Thresholds in the tolerable EF have historically been projected using ordinary least squares (OLS) fits of power law exponents for the simple $0 \mathrm{D}$ parameters shown in Section 2. The fit itself is simply a least 

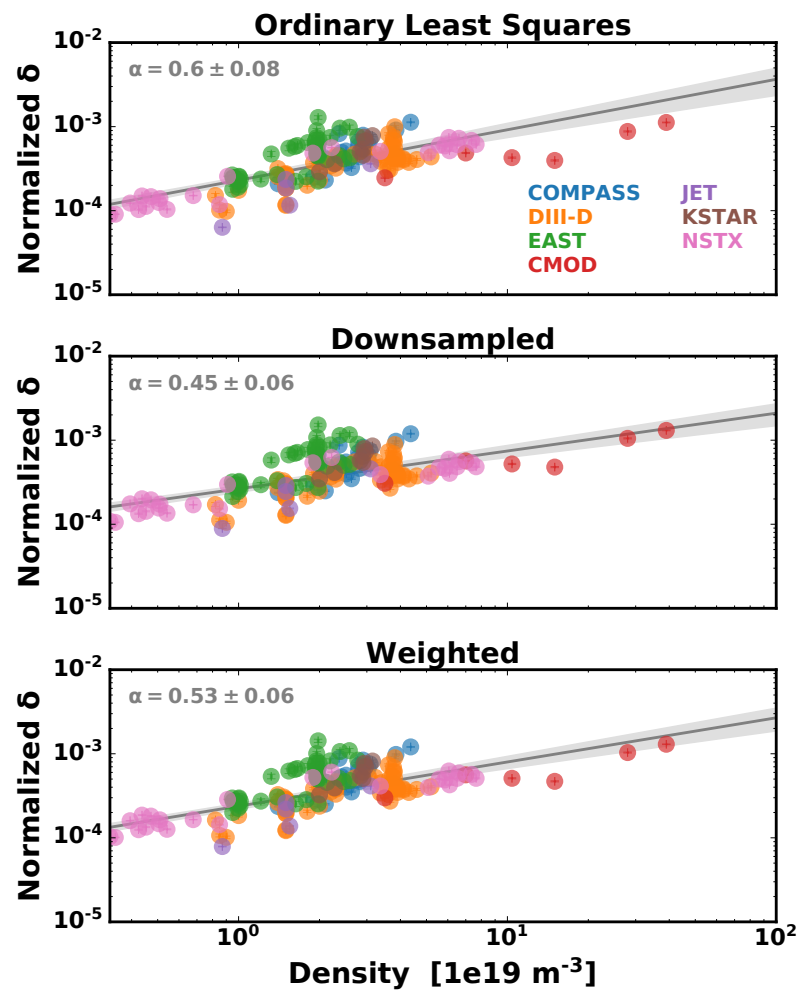

Figure 3. Empirical EF thresholds normalized by $\left[B_{T}^{\alpha_{B}} R_{0}^{\alpha_{R}}\left(\beta_{n} / \ell_{i}\right)^{\alpha_{\beta}}\right]$ as a function of density for the ordinary least squares (top), downsampled least squares (middle) and weighted least squares (bottom) regression. In each case, a grey line shows the fit density scaling, and all other $\alpha$ 's can be found in Table 1.

squares solution of the linear equation,

$$
\mathbf{D} \cdot \boldsymbol{\alpha}=\boldsymbol{\delta}
$$

where the rows of the data matrix D are filled with the parameters shown in figure 1 ,

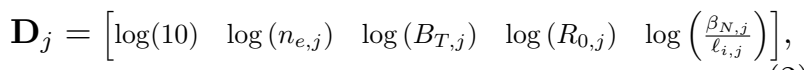

the power law exponents vectors $\boldsymbol{\alpha}$ contains the unknown power law exponents corresponding to the constant and each scaling parameter, $\left\{\alpha_{c}, \alpha_{n}, \alpha_{B}, \alpha_{R}, \alpha_{\beta}\right\}$, and the threshold vector contains the $\log$ overlap thresholds, $\log \left(\delta_{j}\right)$, determined experimentally.

Historically, the unknown scaling coefficients have been fit using simple least squares fitting. This is motivated in part by power scalings that can be derived from reduced models in distinct parameter regimes [21-24]. This implicitly assumes that the power-law scaling model is a true description of the system, and that deviations from the scaling are Gaussian distribution errors. Although the underlying ordering assumptions of individual theoretical scalings are often violated even within a single machine and/or single-parameter scans, least-squares power scaling fits have been used extensively to compare experimental observations to the reduced resistive layer models [79, 12-20]. The primary goal of a multi-machine, multi-parameter scaling is not to validate a particular reduced model, but rather to provide a rough estimate of critical plasma properties at the design points of new machines. Such an empirical scaling, spanning large parameter ranges for which there is no single theoretical power scaling model, have been invaluable tools for the fusion community [33, 38, 39].

One common issue for such tokamak scaling studies using such linear regression techniques is the possibility of co-linearity in the data [38]. The colinearity of the data used in the EF threshold scaling can be inspected visually in figure 1 . The condition number (ratio of largest to smallest eigenvalues) of a normalized fit matrix $\tilde{\mathbf{D}}$ (wherein columns of independent variable spans have unit length) quantify the co-linearity of the fit, with values of 20 or greater generally being considered problematic [40, 41]. A simple, least-squares regression of the the full database shown in figure 1 is given in table 1 . The condition number of the full database regression is 7.0. A fit of a reduced database, excluding $\mathrm{H}$-mode data (but including the $4 \mathrm{~L}$-mode plasmas), is also provided as most historically quoted thresholds have not included $\mathrm{H}$-mode data. The condition number of this fit is 8.6. These values are well below 20, confirming the fits are robust to issues of co-linearity.

The biases introduced by vastly different sampling in different machines is also always a concern for such scalings as well. This has been addressed in confinement scalings previously by performing two step regressions, performed first on individual machines before being aggregated [39]. Here, we employ a simple Monte-Carlo technique: performing the regression on sub-sets of data randomly downsampled to 6 points per machine. The results of 300 of such randomly downsampled ordinary least squares (DSOLS) regressions are presented in Table 1 with values and uncertainties reflecting the means and standard deviations of the individual scaling exponents and projections. We can see the exponents remain mostly consistent with the OLS fits, with the exception of the low value and high uncertainty pressure scaling coefficient. Importantly, the final projection to ITER, remain close to the OLS prediction that ITER can withstand EF overlap values on the order of $10^{-4}$.

\subsection{Weighted Regression}

The high cost of tokamak experiments means that it is an expensive waste to downsample available data in an attempt to even the distribution of empirical points. As an alternate approach, we can use a weighting 
scheme that enables use of all the available data while obtaining fits that describe the broadest trends across the irregularly sampled machines distributed throughout the parameter space.

Here, we use weighted least squares (WLS) regressions to investigate and remove the sampling biases of our empirical database and obtain reliable projections to new machines such as ITER. The weight of each empirical point is inversely proportional to the probability density function (PDF) at that point calculated using a multivariate $\mathrm{KDE}$ in the normalized log space shown in figure 1 . The KDE is calculated using product Gaussian kernels calculated using the statsmodels python package [41]. The results reported here use cross-validation maximum likelihood bandwidths that maximize the leave-oneout likelihood $[42,43]$. These correspond to 0.13 , $0.05,0.001$, and 0.18 for the $n_{e}, B_{T}, R_{0}$, and $\beta_{N} / \ell_{i}$ terms respectively in the full database regression. The conclusions drawn here are not sensitive to this choice however, and the bandwidths can be varied by nearly an order of magnitude while maintaining overlap with the reported uncertainties. This provides a continuous estimate of the PDF from the data, used to weight unique data where the sampling is sparse more than the data where the density is high in a heavily sampled parameter space.

The inverse PDF weighting preferentially values matching data far from other data in the parameter space and devalues matching data in densely populated parametric ranges (i.e. the wealth of data from DIII-D, EAST, and KSTAR near $R_{0} \approx 1.7, n_{e} \approx$ 2). This is done to better describe the broadest trends across the sampled domain and thus provide the most reasonable projections outside the edges of that domain. This provides an alternate way of investigating the potential for sampling biases from the different machines. However, it may not always be the "best" fit. The use of least squares still implicitly assume the applicability of a power scaling model across the full data set, which the theoretical scalings tell us have distinct scaling in various parameter regimes [21-24] and local scalings or interpolation methods might more accurately predict new thresholds within the well studied regimes. One might also imagine this choice of weighting skew projections to high(low) values in one parameter $p_{i}$ if a small number of points lie in a novel regime of low(high) $p_{i}$ inconsistent with an otherwise regular trend. It is taken as a reasonable choice in this case because it weights more heavily the sparse data nearest the ITER parameters of primary interest for EF scaling projections.

Figure 2 and Figure 3 show how this principle impacts the fit power scaling description of the existing database. Each figure shows results of a full multidevice, multi-parameter regression of the form shown in Eq. (1). Each panel is similar to a component and component-plus-residual plot, showing the penetration threshold $\delta$ normalized by all the scalings $p_{i}^{\alpha_{i}}$ for parameters $p_{i}$ not shown on the x-axis. The y-axis of Figure 2, for instance, is $\delta /\left[n_{e}^{\alpha_{n}} R_{0}^{\alpha_{R}}\left(\beta_{n} / \ell_{i}\right)^{\alpha_{\beta}}\right]$. The plots in this figure show cases for which a OLS regressiom was used to perform the regression (top) and a case where a WLS regression was used (bottom). The plots show how the unweighted fit describes the dense cloud of data between 1 and $2 T$ well at the cost of large discrepancies with the uniquely high field C-MOD data. The weighted regression scaling better describes the breadth of the data across the full domain (spanning more than an order of magnitude in toroidal field), accurately reproducing the high field C-MOD thresholds. Figure 3, for which the y-axis is $\delta /\left[B_{T}^{\alpha_{B}} R_{0}^{\alpha_{R}}\left(\beta_{n} / \ell_{i}\right)^{\alpha_{\beta}}\right]$, shows a similar result in which the weighted fit more faithfully represents the the C-MOD points at the highest densities. The downsampled regressions also capture the JET cases, as they effectively increase the weight of the JET data compared to the highly downsampled DIII-D and EAST data. Note, when examining these figures for a sense of overall fit accuracy, that the variance evident in Figure 3 may be an underestimate of the true variance due to correlations with the normalized pressure. For a full picture of the multi-parameter fit residuals, please refer to Figure 2 in Ref. [27].

The full set of weighted least squares regression results for the $n=1 \mathrm{EF}$ threshold scaling exponents can be compared to corresponding OLS regression exponents in Table 1. In neither case are the scalings drastically altered by the weighting. The weighting procedure does not cause any departure from the expected physics, maintaining consistency with the previously documented theoretical and single-device, single-parameter scalings $[7-9,12-24,26,27]$. The largest differences with the OLS fits apear in the pressure scaling of the Ohmic and L-mode database, which is a relatively weak dependence with relatively high uncertainty in all cases. The density scaling, which is the most thoroughly documented $[7-9,12-20]$ is practically unaffected. This is reassuring, as is the fact that only minor adjustments to the other scalings are sufficient to better describe the experiments like CMOD that are expanding the edges of the database's domain.

\section{Conclusions}

This paper details the distribution of the international database of error field penetration threshold data and provides a reference for the simple least-squares 


\begin{tabular}{|l|c|c|c|c|c|c|c|}
\hline Data & Fit & $\alpha_{c}$ & $\alpha_{n}$ & $\alpha_{B}$ & $\alpha_{R}$ & $\alpha_{\beta}$ & $10^{4} \delta_{\text {pen }}^{\text {ITER }}$ \\
\hline O,L & OLS & $-3.57 \pm 0.05$ & $+0.63 \pm 0.09$ & $-0.98 \pm 0.12$ & $+0.15 \pm 0.08$ & $-0.13 \pm 0.1$ & $2.71 \pm 1.31$ \\
O,L & DSOLS & $-3.39 \pm 0.06$ & $+0.58 \pm 0.08$ & $-1.08 \pm 0.10$ & $+0.19 \pm 0.07$ & $+0.26 \pm 0.10$ & $4.30 \pm 0.91$ \\
O,L & WLS & $-3.46 \pm 0.05$ & $+0.64 \pm 0.06$ & $-1.14 \pm 0.08$ & $+0.20 \pm 0.07$ & $+0.15 \pm 0.07$ & $3.54 \pm 0.54$ \\
\hline O,L,H & OLS & $-3.64 \pm 0.04$ & $+0.60 \pm 0.08$ & $-0.95 \pm 0.08$ & $+0.12 \pm 0.08$ & $-0.30 \pm 0.05$ & $1.93 \pm 0.84$ \\
O,L,H & DSOLS & $-3.58 \pm 0.04$ & $+0.45 \pm 0.06$ & $-0.94 \pm 0.08$ & $+0.09 \pm 0.07$ & $-0.15 \pm 0.05$ & $1.66 \pm 0.20$ \\
O,L,H & WLS & $-3.62 \pm 0.04$ & $+0.53 \pm 0.06$ & $-0.95 \pm 0.07$ & $+0.14 \pm 0.08$ & $-0.19 \pm 0.05$ & $1.87 \pm 0.23$ \\
\hline
\end{tabular}

Table 1. Scaling exponents from ordinary least squares (OLS), KDE weighted least squares (WLS) and downsampled OLS (DSOLS) regressions using the present MDC-19 databases for $n=1$ dominant mode overlap EF thresholds. The last column shows the corresponding threshold projection to ITER.

scalings used to project thresholds to ITER. The key conclusions of the database distribution study, clarified by the use of kernel density estimations, is that major opportunities for new data to strengthen the database exist. Of course, more data mined from past experiments on uniquely positioned machines such as C-MOD would be of particular interest and could result in better conditioned fits. The density distributions shown here also highlight key gaps in the database that can be filled by near-future experiments. One such gap is found in the toroidal field values between NSTX and DIII-D. There is an opportunity for NSTX-U to fill this gap soon when it begins operation. Similarly, there is an opportunity for future compact, high-field devices to supplement the limited data near the high ITER field values. In addition to the opportunities for new devices, the database distribution clearly reveals that there is a valuable opportunity for all existing devices to contribute meaningful data to this ITPA effort in high normalized pressure plasma scenarios.

The bottom line result of the scalings presented here is that the projection of the EF threshold to our chosen ITER scenario is robustly on the order of $\delta \approx 10^{-4}$. Averaging the methods reported in Table 1 gives values of $1.82 \pm 0.30$ and $3.50 \pm 0.60$ for regressions with and without $\mathrm{H}$-modes respectively. Using a WLS regression does tend to slightly modify the ITER projected threshold, but not beyond overlapping error bars. The DSOLS projection excluding $\mathrm{H}$-mode data does vary more significantly, but is still within a factor of two of the simple OLS projection. All such projected values are consistent with the order of magnitude experienced in current devices and with previous ITER estimates [25]. This level of EF tolerance is well understood and has been safely taken into account by the combination of ITER engineering tolerances and error field correction coil design [31, 32].

This work provides confidence in the simple, four parameter regression's robustness to co-linearity and sampling biases while reconfirming the validity of the existing ITER EF correction strategy. The robustness of the scalings to the uneven machine sampling in particular, opens additional opportunities for the community to revisit the wealth of experiments that are not expressly EF threshold studies but did drive core tearing modes when applying resonant magnetic perturbations. This data, if found, could safely be added to the multi-device database without worry of "swamping" more stringently limited data from other machines and may further improve our confidence in the regression.

While this work introduces the critical features of the EF threshold database and basic scalings, opportunities to improve the rigour of EF threshold predictions certainly still exist. There may be applications predicting thresholds in new machines or scenarios within the well populated range of the database for example, that could avoid the assertion that there is one global trend valid for all the device using more sophisticated non-parametric kernel regression techniques [41, 44]. Within the stated objective of a broad scaling similar to confinement scalings, future work could further investigate more advanced statistical methods, perhaps minimizing the $L^{\infty}$ norm to minimize the maximum deviation. This paper serves as only an initial identification of the sampling bias and its basic compensation with the expectation that regression methods will continue to improve as the available data continues to expand.

\section{Acknowledgements}

This work was supported by the U.S. Department of Energy Office of Science Office of Fusion Energy Sciences using the DIII-D National Fusion Facility and Alcator C-Mod, both DOE Office of Science user facilities, under Awards DE-FC02-04ER54698, DE- AC0209CH11466 and DE-FC02-99ER54512. The work was also supported by the National Key R\&D Program of China under Grant No. 2017YFE0301100, the 
Czech Science Foundation (GA CR) under the grant number 19-15229S, and by MEYS of CR projects number 8D15001 and LM2015045. The work has been carried out within the framework of the project COMPASS-U: Tokamak for cutting-edge fusion research (No. CZ.02.1.01/0.0/0.0/16_019/0000768) and co-funded from European structural and investment funds. This work has been carried out within the framework of the EUROfusion Consortium and has received funding from the Euratom research and training program 2014-2018 and 2019-2020 under grant agreement No 633053. The views and opinions expressed herein do not necessarily reflect those of the European Commission

Disclaimer: This report was prepared as an account of work sponsored by an agency of the United States Government. Neither the United States Government nor any agency thereof, nor any of their employees, makes any warranty, express or implied, or assumes any legal liability or responsibility for the accuracy, completeness, or usefulness of any information, apparatus, product, or process disclosed, or represents that its use would not infringe privately owned rights. Reference herein to any specific commercial product, process, or service by trade name, trademark, manufacturer, or otherwise does not necessarily constitute or imply its endorsement, recommendation, or favoring by the United States Government or any agency thereof. The views and opinions of authors expressed herein do not necessarily state or reflect those of the United States Government or any agency thereof.

\section{References}

${ }^{1}$ R. J. La Haye, R. Fitzpatrick, T. C. Hender, a. W. Morris, J. T. Scoville, and T. N. Todd, "Critical error fields for locked mode instability in tokamaks", Physics of Fluids B: Plasma Physics 4, 2098 (1992).

${ }^{2}$ R. J. La Haye and J. T. Scoville, "A method to measure poloidal field coil irregularities in toroidal plasma devices", Review of Scientific Instruments 62, 2146-2153 (1991).

${ }^{3}$ A. Garofalo, R. L. Haye, and J. Scoville, "Analysis and correction of intrinsic non-axisymmetric magnetic fields in high- DIII-D plasmas", Nuclear Fusion 42, 1335-1339 (2002).

${ }^{4}$ J. Luxon, M. Schaffer, G. Jackson, J. Leuer, A. Nagy, J. Scoville, and E. Strait, "Anomalies in the applied magnetic fields in DIII-D and their implications for the understanding of stability experiments", en, Nuclear Fusion 43, 1813-1828 (2003).
${ }^{5}$ J.-K. Park, M. J. Schaffer, R. J. La Haye, T. J. Scoville, and J. E. Menard, "Error field correction in DIII-D Ohmic plasmas with either handedness", Nuclear Fusion 51, 023003 (2011).

${ }^{6}$ H.-H. Wang, Y.-W. Sun, J.-P. Qian, T.-H. Shi, B. Shen, S. Gu, Y.-Q. Liu, W.-F. Guo, N. Chu, K.-Y. He, M.-N. Jia, D.-L. Chen, M.-M. Xue, J. Ren, Y. Wang, Z.-C. Sheng, B.-J. Xiao, Z.-P. Luo, Y. Liu, H.-Q. Liu, H.-L. Zhao, L. Zeng, X.-Z. Gong, Y.-F. Liang, and B.-N. Wan, "Observation of spectrum effect on the measurement of intrinsic error field on EAST", Nuclear Fusion 56, 066011 (2016).

${ }^{7}$ S. M. Wolfe, I. H. Hutchinson, R. S. Granetz, J. Rice, A. Hubbard, A. Lynn, P. Phillips, T. C. Hender, D. F. Howell, R. J. La Haye, and J. T. Scoville, "Nonaxisymmetric field effects on Alcator C-Mod", Physics of Plasmas 12, 056110 (2005).

${ }^{8}$ R. Buttery, M. D. Benedetti, T. Hender, and B. Tubbing, "Error field experiments in JET", en, Nuclear Fusion 40, 807-819 (2000).

${ }^{9}$ J. Menard, R. Bell, D. Gates, S. Gerhardt, J.-K. Park, S. Sabbagh, J. Berkery, A. Egan, J. Kallman, S. Kaye, B. LeBlanc, Y. Liu, A. Sontag, D. Swanson, H. Yuh, and W. Zhu, "Progress in understanding error-field physics in NSTX spherical torus plasmas", Nuclear Fusion 50, 045008 (2010).

${ }^{10}$ M. Maraschek, S. Fietz, A. Gude, S. Günter, R. Koslowski, K. Lackner, K. Lüders, T. Lunt, G. Pautasso, E. Strumberger, W. Suttrop, Q. Yu, H. Zohm, and A. U. Team, "Measurement and impact of the $\mathrm{n}=1$ intrinsic error field at ASDEX Upgrade", 40th EPS Conference on Plasma Physics, P4.127 (2013).

${ }^{11}$ Y. In, J. Park, J. Jeon, J. Kim, and M. Okabayashi, "Extremely low intrinsic non-axisymmetric field in KSTAR and its implications", Nuclear Fusion 55, 043004 (2015).

${ }^{12}$ J. Scoville, R. La Haye, A. Kellman, T. Osborne, R. Stambaugh, E. J. Strait, and T. Taylor, "Locked modes in DIII-D and a method for prevention of the low density mode", en, Nuclear Fusion 31, 875-890 (1991).

${ }^{13}$ R. Buttery, M. D. Benedetti, D. Gates, Y. Gribov, T. Hender, R. L. Haye, P. Leahy, J. Leuer, A. Morris, A. Santagiustina, J. Scoville, B. Tubbing, J. Team, C.-D. R. Team, and D.-D. Team, "Error field mode studies on JET, COMPASS-D and DIII-D, and implications for ITER", Nuclear Fusion 39, 18271835 (1999). 
${ }^{14}$ R. Wolf, W. Biel, M. d. Bock, K. Finken, S. Günter, G. Hogeweij, S. Jachmich, M. Jakubowski, R. Jaspers, A. Krämer-Flecken, H. Koslowski, M. Lehnen, Y. Liang, B. Unterberg, S. Varshney, M. v. Hellermann, Q. Yu, O. Zimmermann, S. Abdullaev, A. Donné, U. Samm, B. Schweer, M. Tokar, E. Westerhof, and t. T. Team, "Effect of the dynamic ergodic divertor in the TEXTOR tokamak on MHD stability, plasma rotation and transport", Nuclear Fusion 45, 1700-1707 (2005).

${ }^{15}$ D. Howell, T. Hender, and G. Cunningham, "Locked mode thresholds on the MAST spherical tokamak", Nuclear Fusion 47, 1336-1340 (2007).

${ }^{16}$ T. Hender, R. Fitzpatrick, A. Morris, P. Carolan, R. Durst, T. Edlington, J. Ferreira, S. Fielding, P. Haynes, J. Hugill, I. Jenkins, R. L. Haye, B. Parham, D. Robinson, T. Todd, M. Valovic, and G. Vayakis, "Effect of resonant magnetic perturbations on COMPASS-C tokamak discharges", Nuclear Fusion 32, 2091-2117 (1992).

${ }^{17}$ E. Lazzaro, R. J. Buttery, T. C. Hender, P. Zanca, R. Fitzpatrick, M. Bigi, T. Bolzonella, R. Coelho, M. DeBenedetti, S. Nowak, O. Sauter, and M. Stamp, "Error field locked modes thresholds in rotating plasmas, anomalous braking and spin-up", Physics of Plasmas 9, 3906-3918 (2002).

${ }^{18}$ J.-K. Park, J. E. Menard, S. P. Gerhardt, R. J. Buttery, S. A. Sabbagh, R. E. Bell, and B. P. LeBlanc, "Sensitivity to error fields in NSTX high $\beta$ plasmas", Nuclear Fusion 52, 023004 (2012).

${ }^{19}$ N. Wang, B. Rao, Q. Hu, Y. Ding, Z. Chen, L. Gao, W. Jin, B. Yi, W. Zeng, Q. Li, Y. Liu, H. Xu, G. Zhuang, and Y. Pan, "Study of the penetration of resonant magnetic perturbations in J-TEXT", Nuclear Fusion 54, 064014 (2014).

${ }^{20}$ H.-H. Wang, Y.-W. Sun, T.-H. Shi, Q. Zang, Y.-Q. Liu, X. Yang, S. Gu, K.-Y. He, X. Gu, J.-P. Qian, B. Shen, Z.-P. Luo, N. Chu, M.-N. Jia, Z.-C. Sheng, H.-Q. Liu, X.-Z. Gong, and B.-N. Wan, "Density scaling on $\mathrm{n}=1$ error field penetration in ohmically heated discharges in EAST", Nuclear Fusion 58, 056024 (2018).

${ }^{21}$ R. Fitzpatrick and T. C. Hender, "The interaction of resonant magnetic perturbations with rotating plasmas", Physics of Fluids B: Plasma Physics 3, 644-673 (1991).

${ }^{22}$ R. Fitzpatrick, "Interaction of tearing modes with external structures in cylindrical geometry (plasma)", Nuclear Fusion 33, 1049-1084 (1993).

${ }^{23}$ J. Scoville and R. L. Haye, "Multi-mode error field correction on the DIII-D tokamak", Nuclear Fusion 43, 250-257 (2003).
${ }^{24}$ R. Fitzpatrick, "Nonlinear error-field penetration in low density ohmically heated tokamak plasmas", Plasma Physics and Controlled Fusion 54, 094002 (2012).

${ }^{25}$ R. J. Buttery, A. H. Boozer, Y. Q. Liu, J.-K. Park, N. M. Ferraro, V. Amoskov, Y. Gribov, R. J. La Haye, E. Lamzin, J. E. Menard, M. J. Schaffer, E. J. Strait, and the DIII-D Team, "The limits and challenges of error field correction for ITER", Physics of Plasmas 19, 056111 (2012).

${ }^{26}$ J.-K. Park, N. Logan, C. Paz-Soldan, T. Markovič, H. H. Wang, Y. In, Y. Q. Liu, N. Ferraro, L. Piron, P. Piovesan, C. Myers, B. A. Grierson, S. R. Haskey, D. M. Orlov, O. Meneghini, and M. Maraschek, "2017 MDC-19 Report: Assessment of Error Field Correction Criteria for ITER", Private Communication: ITER report, ITER_D_UML (2017).

${ }^{27}$ N. Logan, J.-K. Park, Q. Hu, C. Paz-Soldan, T. Markovic, H.-H. Wang, Y. In, L. Piron, P. Piovesan, C. E. Myers, M. Maraschek, S. M. Wolfe, E. J. Strait, and S. Munaretto, "Empirical scaling of the $\mathrm{n}=2$ error field penetration threshold in tokamaks", Nuclear Fusion (2020).

${ }^{28}$ J.-K. Park, A. H. Boozer, J. E. Menard, and M. J. Schaffer, "Error field correction in ITER", en, Nuclear Fusion 48, 045006 (2008).

${ }^{29}$ C. Paz-Soldan, M. J. Lanctot, N. C. Logan, D. Shiraki, R. J. Buttery, J. M. Hanson, R. J. La Haye, J.-K. Park, W. M. Solomon, and E. J. Strait, "The importance of matched poloidal spectra to error field correction in DIII-D", Physics of Plasmas 21, 072503 (2014).

${ }^{30}$ N. Logan, C. Paz-Soldan, J.-K. Park, and R. Nazikian, "Identification of multi-modal plasma responses to applied magnetic perturbations using the plasma reluctance", Physics of Plasmas 23 (2016).

${ }^{31}$ V. Amoskov, A. Belov, V. Belyakov, O. Filatov, Y. Gribov, E. Lamzin, N. Maximenkova, B. Mingalev, and S. Sytchevsky, "Statistical analysis of expected error fields in tokamaks and their correction", Plasma Devices and Operations 13, 87-103 (2005).

${ }^{32}$ V. Amoskov, Y. Gribov, E. Lamzin, and S. Sytchevsky, "Assessment of $\mathrm{n}=1$ 'overlap' error field produced by localized steel objects placed at different levels of ITER Tokamak building", Fusion Engineering and Design 148, 111271 (2019).

${ }^{33}$ R. J. Goldston, "Energy confinement scaling in Tokamaks: some implications of recent experiments with Ohmic and strong auxiliary heating", Plasma Physics and Controlled Fusion 26, 87-103 (1984). 
${ }^{34}$ V. Amoskov, A. Belov, V. Belyakov, O. Filatov, Y. Gribov, E. Lamzin, N. Maximenkova, B. Mingalev, and S. Sytchevsky, "Fourier analysis of 3D error fields in tokamaks", Plasma Devices and Operations 12, 285-298 (2004).

${ }^{35}$ T. Hender, J. Wesley, J. Bialek, A. Bondeson, A. Boozer, R. Buttery, A. Garofalo, T. Goodman, R. Granetz, Y. Gribov, O. Gruber, M. Gryaznevich, G. Giruzzi, S. Günter, N. Hayashi, P. Helander, C. Hegna, D. Howell, D. Humphreys, G. Huysmans, A. Hyatt, A. Isayama, S. Jardin, Y. Kawano, A. Kellman, C. Kessel, H. Koslowski, R. L. Haye, E. Lazzaro, Y. Liu, V. Lukash, J. Manickam, S. Medvedev, V. Mertens, S. Mirnov, Y. Nakamura, G. Navratil, M. Okabayashi, T. Ozeki, R. Paccagnella, G. Pautasso, F. Porcelli, V. Pustovitov, V. Riccardo, M. Sato, O. Sauter, M. Schaffer, M. Shimada, P. Sonato, E. Strait, M. Sugihara, M. Takechi, A. Turnbull, E. Westerhof, D. Whyte, R. Yoshino, H. Zohm, D. Group the ITPA MHD, and Magnet, "Progress in the ITER Physics Basis Chapter 3: MHD stability, operational limits and disruptions", Nuclear Fusion 47, S128 (2007).

${ }^{36}$ J. Knaster, V. Amoskov, A. Formisano, Y. Gribov, E. Lamzin, L. Martone, N. Maximenkova, N. Mitchell, A. Portone, S. Sytchevsky, and P. Testoni, "ITER non-axisymmetric error fields induced by its magnet system", Fusion Engineering and Design 86, 10531056 (2011).

${ }^{37}$ F. Poli, E. Fredrickson, M. Henderson, S.-H. Kim, N. Bertelli, E. Poli, D. Farina, and L. Figini, "Electron cyclotron power management for control of neoclassical tearing modes in the ITER baseline scenario", Nuclear Fusion 58, 016007 (2018).

${ }^{38}$ S. Kaye, M. Greenwald, U. Stroth, O. Kardaun, A. Kus, D. Schissel, J. DeBoo, G. Bracco, K. Thomsen, J. Cordey, Y. Miura, T. Matsuda, H. Tamai, T. Takizuda, T. Hirayama, H. Kikuchi, O. Naito, A. Chudnovskij, J. Ongena, and G. Hoang, "ITER L mode confinement database", Nuclear Fusion 37, 1303-1328 (1997).

${ }^{39}$ S. Kaye and R. Goldston, "Global energy confinement scaling for neutral-beam-heated tokamaks", Nuclear Fusion 25, 65 (1985).

${ }^{40} \mathrm{~W}$. H. Greene, Econometric Analysis, 5th ed. (Prentice Hall, Upper Saddle River, New Jersey, June 2002).

${ }^{41} \mathrm{~S}$. Seabold and J. Perktold, "Statsmodels: Econometric and Statistical Modeling with Python", in Proc. of the 9th python in science conf (2010), p. 57.

${ }^{42}$ J. S. Racine, "Nonparametric Econometrics: A Primer", Foundations and Trends $\AA$ in Econometrics 3, 1-88 (2008).
${ }^{43}$ B. W. Silverman, "Density Estimation for Statistics and Data Analysis", Monographs on Statistics and Applied Probability (1986).

${ }^{44}$ T. Hastie, R. Tibshirani, and J. Friedman, The Elements of Statistical Learning: Data Mining, Inference, and Prediction, Second Edi (SpringerVerlag, New York, 2009). 